\title{
CONVERGENCE OF SOLUTIONS OF TIME-VARYING LINEAR SYSTEMS WITH INTEGRABLE FORCING TERM
}

\section{JITSURO SUGIE}

\author{
(Received 27 February 2008)
}

\section{Abstract}

The following system is considered in this paper:

$$
x^{\prime}=-e(t) x+f(t) y, \quad y^{\prime}=-g(t) x-h(t) y+p(t) .
$$

The primary goal is to establish conditions on time-varying coefficients $e(t), f(t), g(t)$ and $h(t)$ and a forcing term $p(t)$ for all solutions to converge to the origin $(0,0)$ as $t \rightarrow \infty$. Here, the zero solution of the corresponding homogeneous linear system is assumed to be neither uniformly stable nor uniformly attractive. Sufficient conditions are given for asymptotic stability of the zero solution of the nonlinear perturbed system

$$
x^{\prime}=-e(t) x+f(t) y, \quad y^{\prime}=-g(t) x-h(t) y+q(t, x, y)
$$

under the assumption that $q(t, 0,0)=0$.

2000 Mathematics subject classification: 34D05, 34D10, 34D20.

Keywords and phrases: nonhomogeneous linear systems, weakly integrally positive, perturbation problems.

\section{Introduction}

Let $A(t)$ and $\mathbf{b}(t)$ be matrix and vector functions, respectively, which are continuous for $t \geq 0$. The asymptotic behavior of solutions of the nonhomogeneous linear system

$$
\mathbf{x}^{\prime}=A(t) \mathbf{x}+\mathbf{b}(t)
$$

has been studied extensively. The main tool in these studies is the variation of constants formula, that is, the solution $\mathbf{x}(t)$ satisfying $\mathbf{x}(\tau)=\xi$ for $\tau \geq 0$ and $\xi \in \mathbb{R}^{n}$ is given by

$$
\mathbf{x}(t)=Y(t) Y^{-1}(\tau) \xi+Y(t) \int_{\tau}^{t} Y^{-1}(s) \mathbf{b}(s) d s,
$$

where $Y(t)$ is a fundamental matrix for the linear system

$$
\mathbf{y}^{\prime}=A(t) \mathbf{y} .
$$

Supported in part by Grant-in-Aid for Scientific Research, No. 19540182.

(C) 2009 Australian Mathematical Society 0004-9727/09 \$A2.00+0.00 
We can express various stabilities of the zero solution in terms of a fundamental matrix for $(L)$. For example, the zero solution of $(L)$ is uniformly asymptotically stable (for the definition, see Section 2) if and only if there exist positive constants $R$ and $\rho$ such that

$$
\left\|Y(t) Y^{-1}(s)\right\| \stackrel{\text { def }}{=} \sup _{\|\mathbf{y}\|=1}\left\|Y(t) Y^{-1}(s) \mathbf{y}\right\| \leq R e^{-\rho(t-s)} \quad \text { for } 0 \leq s \leq t \leq \infty,
$$

where $\|\mathbf{y}\|$ is an arbitrary norm of a vector $\mathbf{y}$. The concept of uniform asymptotic stability is a combination of the concepts of uniform stability and uniform attractivity. Using the inequality (1.1) with the variation of constants formula, we can easily verify that if the zero solution of $(L)$ is uniformly asymptotically stable and if

$$
\int_{0}^{\infty}\|\mathbf{b}(s)\| d s<\infty
$$

then every solution $\mathbf{x}(t)$ of $(N)$ tends to $\mathbf{0} \in \mathbb{R}^{n}$ as $t \rightarrow \infty$. However, we cannot derive this conclusion from uniform stability and attractivity of the zero solution of $(L)$, instead of uniform asymptotic stability. This means that uniform attractivity for the system $(L)$ is essential for proving the convergence of solutions of $(N)$.

Many attempts have been made to alleviate the perturbation problem. For instance, Strauss and Yorke [10] have presented some results on the preservation of uniform stability from system $(L)$ to system $(N)$ without requiring uniform attractivity of the zero solution of $(L)$. One of their results is that if the zero solution of $(L)$ is uniformly stable and attractive, then condition (1.2) is necessary and sufficient for the origin $\mathbf{0}$ to be eventually uniformly stable and eventually attractive for system $(N)$. The concepts of eventual uniform stability and eventual attractivity are weaker than those of uniform stability and attractivity, respectively (for details, see [10]). Note that there is a case in which the origin is unstable even if it is eventually uniformly stable for system $(N)$.

In their result, it is natural to consider the origin $\mathbf{0}$ instead of the zero solution, because system $(N)$ does not have the zero solution. Of course, if the origin $\mathbf{0}$ is eventually attractive, then for a sufficiently large $\tau$ and a sufficiently small $\|\xi\|$, every solution $\mathbf{x}(t)$ of $(N)$ satisfying $\mathbf{x}(\tau)=\xi$ tends $\mathbf{0}$ to as $t \rightarrow \infty$. However, we can only conclude the eventual convergence of solutions of $(N)$.

The following question then arises. What kind of condition on $A(t)$ will guarantee the convergence of all solutions of $(N)$ under the assumption that the zero solution of $(L)$ is uniformly stable and attractive? The attractivity is not assumed to be uniform.

Strauss and Yorke [10] have also given an example in which the origin $\mathbf{0}$ is neither eventually stable nor eventually attractive for system $(N)$, even though the zero solution of $(L)$ is stable and attractive, and condition (1.2) holds. Hence, in their result, we cannot replace 'uniform stability' by 'stability' of the zero solution of $(L)$.

Another question now arises. What kind of condition on $A(t)$ will guarantee the convergence of all solutions of $(N)$ under the assumption that the zero solution of $(L)$ is stable and attractive? Neither the attractivity nor the stability is assumed to be uniform. 
We answer the above question in Section 2. In Section 3, we attempt a generalization of our results. To this end, we consider the perturbed system

$$
\mathbf{x}^{\prime}=A(t) \mathbf{x}+\mathbf{f}(t, \mathbf{x})
$$

where $\mathbf{f}$ is a continuous vector function, and we examine perturbation problems on the convergence of solutions of $(P)$. For the case in which $\mathbf{f}(t, \mathbf{0})=\mathbf{0}$, system $(P)$ has the zero solution. Coppel [1, Theorem 6, p. 64] has given a result on uniform stability and asymptotic stability of the zero solution of $(P)$. We compare the present results with Coppel's result. To clarify the difference between the two results, we also give a number of examples.

\section{Nonhomogeneous system}

Consider the linear system

$$
x^{\prime}=-e(t) x+f(t) y, \quad y^{\prime}=-g(t) x-h(t) y,
$$

where the components $f(t)$ and $g(t)$ are continuous for $t \geq 0$ and the components $e(t)$ and $h(t)$ are piecewise continuous for $t \geq 0$. It is clear that system (2.1) has the zero solution $(x(t), y(t)) \equiv(0,0)$.

We denote the solution of (2.1) through $\left(t_{0}, x_{0}, y_{0}\right)$ by $\left(x\left(t ; t_{0}, x_{0}, y_{0}\right)\right.$, $\left.y\left(t ; t_{0}, x_{0}, y_{0}\right)\right)$. The zero solution of $(2.1)$ is said to be stable, if for any $t_{0} \geq 0$ and any $\alpha>0$, there exists a $\beta\left(t_{0}, \alpha\right)>0$ such that $\left|x_{0}\right|+\left|y_{0}\right|<\beta$ implies $\left|x\left(t ; t_{0}, x_{0}, y_{0}\right)\right|+\left|y\left(t ; t_{0}, x_{0}, y_{0}\right)\right|<\alpha$ for all $t \geq t_{0}$. The zero solution is uniformly stable if it is stable and $\beta$ can be chosen independent of $t_{0}$. The zero solution is said to be attractive if there exists a $\beta_{0}\left(t_{0}\right)>0$ such that $\left|x_{0}\right|+\left|y_{0}\right|<\beta_{0}$ implies $\left|x\left(t ; t_{0}, x_{0}, y_{0}\right)\right|+\left|y\left(t ; t_{0}, x_{0}, y_{0}\right)\right| \rightarrow 0$ as $t \rightarrow \infty$. The zero solution is uniformly attractive if $\beta_{0}$ in the definition of attractivity can be chosen independent of $t_{0}$, and for every $\eta>0$ there is a $T(\eta)>0$ such that $t_{0} \geq 0$ and $\left|x_{0}\right|+\left|y_{0}\right|<\beta_{0}$ imply $\left|x\left(t ; t_{0}, x_{0}, y_{0}\right)\right|+\left|y\left(t ; t_{0}, x_{0}, y_{0}\right)\right|<\eta$ for $t \geq t_{0}+T(\eta)$. The zero solution is said to be asymptotically stable if it is stable and attractive. The zero solution is uniformly asymptotically stable if it is uniformly stable and is uniformly attractive.

DEFINITION 2.1. A nonnegative function $\phi$ is said to be integrally positive if

$$
\int_{I} \phi(t) d t=\infty
$$

for every set $I=\bigcup_{n=1}^{\infty}\left[\tau_{n}, \sigma_{n}\right]$ such that $\tau_{n}<\sigma_{n}<\tau_{n+1}$ and $\sigma_{n}-\tau_{n} \geq \delta>0$. If, in addition, the set $I$ satisfies $\tau_{n+1} \leq \sigma_{n}+\Delta$ for some $\Delta>0$, the function $\phi$ is said to be weakly integrally positive. 
We can cite $1 /(1+t)$ or $\sin ^{2} t /(1+t)$ as a function that is weakly integrally positive, but not integrally positive (refer to [4-6]).

Throughout this paper, we assume that $f(t) g(t)>0$ and $g(t) / f(t)$ is differentiable for $t \geq 0$. Then, we may define

$$
\psi(t)=2(h(t)-e(t))+\frac{f(t)}{g(t)}\left(\frac{g(t)}{f(t)}\right)^{\prime} .
$$

For the sake of brevity, we write

$$
\psi_{+}(t)=\max \{0, \psi(t)\} \quad \text { and } \quad \psi_{-}(t)=\max \{0,-\psi(t)\} .
$$

Recently, the present author has reported the following result [11].

Theorem A. Suppose that:

(i) $E(t) \stackrel{\text { def }}{=} \int_{0}^{t} e(s) d s$ is bounded from below;

(ii) $f(t) \exp (E(t))$ and $g(t) / \exp (E(t))$ are bounded, and $f(t) g(t)>0$ for $t \geq 0$ and $\lim \inf _{t \rightarrow \infty} f(t) g(t)>0$;

(iii) $h(t)$ is bounded;

(iv) $\psi_{+}(t)$ is weakly integrally positive;

(v) $\int_{0}^{\infty} \psi_{-}(s) d s<\infty$.

Then, the zero solution of (2.1) is asymptotically stable. If, in addition, $E(t)$ is bounded from above, then the zero solution of (2.1) is uniformly stable.

Note that the assumptions of Theorem A do not imply that the zero solution is uniformly asymptotically stable. For example, consider system $(2.1)$ with $e(t)=0$, $f(t)=g(t)=1$ and $h(t)=2 /(1+t)$. Then, $\psi(t)=4 /(1+t)$. We can easily confirm that assumptions (i)-(v) in Theorem A are satisfied and $E(t)$ is bounded from above. In this case, a fundamental matrix for system (2.1) is

$$
Y(t)=\left(\begin{array}{cc}
\frac{\sin t}{1+t} & \frac{\cos t}{1+t} \\
\frac{\cos t}{1+t}-\frac{\sin t}{(1+t)^{2}} & -\frac{\sin t}{1+t}-\frac{\cos t}{(1+t)^{2}}
\end{array}\right) .
$$

From a classical result on a fundamental matrix (see, for example, [1, Theorem 1, p. 54]), we can judge that the zero solution is uniformly stable and asymptotically stable, but it is not uniformly asymptotically stable.

Let us add the forcing term $p(t)$ to the second equation of (2.1) and examine the asymptotic behavior of solutions of the nonhomogeneous linear system

$$
x^{\prime}=-e(t) x+f(t) y, \quad y^{\prime}=-g(t) x-h(t) y+p(t) .
$$

We intend to discuss whether all solutions of (2.2) decay as $t$ increases.

Before giving our result, we present some lemmas. 
Lemma 2.2. Assumption (ii) in Theorem A implies the inequalities

$$
0<k \leq \frac{f(t)}{g(t)} e^{2 E(t)} \leq K \quad \text { for } t \geq 0 .
$$

PROOF. Owing to (ii), there exist constants $c_{1}>0$ and $c_{2}>0$ such that

$$
f(t) g(t) \geq c_{1} \quad \text { for } t \geq 0
$$

and

$$
\frac{|g(t)|}{e^{E(t)}} \leq c_{2} \quad \text { for } t \geq 0
$$

Hence, we obtain

$$
\frac{f(t)}{g(t)} e^{2 E(t)}=\frac{f(t) g(t)}{g^{2}(t) / e^{2 E(t)}} \geq \frac{c_{1}}{c_{2}^{2}} \text { for } t \geq 0 .
$$

Again, from (ii), we see that there exists a $c_{3}>0$ with

$$
|f(t)| e^{E(t)} \leq c_{3} \quad \text { for } t \geq 0 .
$$

Moreover, we can select a number $c_{4}>0$ satisfying

$$
\frac{|g(t)|}{e^{E(t)}} \geq c_{4} \quad \text { for } t \geq 0 .
$$

In fact, if there exists a sequence $\left\{\omega_{n}\right\}$ tending to $\infty$ such that

$$
\frac{\left|g\left(\omega_{n}\right)\right|}{e^{E\left(\omega_{n}\right)}} \rightarrow 0 \quad \text { as } n \rightarrow \infty
$$

then, by (2.4),

$$
\left|f\left(\omega_{n}\right)\right| e^{E\left(\omega_{n}\right)} \rightarrow \infty \quad \text { as } n \rightarrow \infty .
$$

This contradicts (2.5). We therefore conclude that

$$
\frac{f(t)}{g(t)} e^{2 E(t)}=\frac{f(t) e^{E(t)}}{g(t) / e^{E(t)}} \leq \frac{c_{3}}{c_{4}} \quad \text { for } t \geq 0 .
$$

Let $k=c_{1} / c_{2}^{2}$ and $K=c_{3} / c_{4}$. Then, we obtain the inequality (2.3).

Lemma 2.3. Suppose that assumption (v) in Theorem A holds. Let $v(t)$ be nonnegative and piecewise continuously differentiable on $\left[t_{0}, \infty\right)$ for some $t_{0} \geq 0$, and let $\phi(t)$ be nonnegative and integrable on $\left[t_{0}, \infty\right)$. If

$$
v^{\prime}(t) \leq \psi_{-}(t) v(t)+\phi(t) \quad \text { for } t \geq t_{0},
$$

then $v^{\prime}(t)$ is absolutely integrable and, therefore, $v(t)$ has a nonnegative limiting value. 
Proof. Since $\psi_{-}(t)$ and $\phi(t)$ are nonnegative and integrable, we can choose nonnegative numbers $A$ and $B$ such that

$$
A=\int_{0}^{\infty} \psi_{-}(s) d s \quad \text { and } \quad B=\int_{0}^{\infty} \phi(s) d s
$$

By (2.6),

$$
\begin{aligned}
v(t) & \leq \exp \left(\int_{t_{0}}^{t} \psi_{-}(s) d s\right)\left(v\left(t_{0}\right)+\int_{t_{0}}^{t} \phi(s) \exp \left(-\int_{t_{0}}^{s} \psi_{-}(\tau) d \tau\right) d s\right) \\
& \leq e^{A}\left(v\left(t_{0}\right)+B\right)
\end{aligned}
$$

for $t \geq t_{0}$. Hence, using (2.6) again, we obtain

$$
v^{\prime}(t) \leq e^{A}\left(v\left(t_{0}\right)+B\right) \psi_{-}(t)+\phi(t) \text { for } t \geq t_{0} .
$$

Since the right-hand side of this inequality is nonnegative for $t \geq t_{0}$, we obtain

$$
v_{+}^{\prime}(t) \leq e^{A}\left(v\left(t_{0}\right)+B\right) \psi_{-}(t)+\phi(t) \quad \text { for } t \geq t_{0} .
$$

Integrating both sides from $t_{0}$ to $\infty$, we obtain

$$
\int_{t_{0}}^{\infty} v_{+}^{\prime}(s) d s \leq e^{A}\left(v\left(t_{0}\right)+B\right) A+B<\infty .
$$

On the other hand, since $v(t) \geq 0$ for $t \geq t_{0}$,

$$
\int_{t_{0}}^{\infty} v_{-}^{\prime}(s) d s \leq v\left(t_{0}\right)+\int_{t_{0}}^{\infty} v_{+}^{\prime}(s) d s<\infty .
$$

We therefore conclude that

$$
\int_{t_{0}}^{\infty}\left|v^{\prime}(s)\right| d s=\int_{t_{0}}^{\infty}\left(v_{+}^{\prime}(s)+v_{-}^{\prime}(s)\right) d s<\infty,
$$

as required. This completes the proof of Lemma 2.3.

LEMMA 2.4. In addition to assumptions (i), (ii) and (v) in Theorem A, if

(vi) $\int_{0}^{\infty}|p(s)| d s<\infty$,

then all solutions of (2.2) are equi-bounded.

ProOF. Define

$$
V(t, x, y)=\frac{1}{2} e^{2 E(t)}\left(x^{2}+\frac{f(t)}{g(t)} y^{2}\right)
$$


on $[0, \infty) \times \mathbb{R}^{2}$ and differentiate $V(t, x, y)$ along any solution of (2.2) obtaining

$$
\dot{V}_{(2.2)}(t, x, y)=-\frac{f(t)}{2 g(t)} e^{2 E(t)} \psi(t) y^{2}+\frac{f(t) p(t)}{g(t)} e^{2 E(t)} y .
$$

Let $P(t)=\int_{0}^{t}|p(s)| d s$. Next, define $W(t, x, y)=e^{-P(t)} V(t, x, y)$ on $[0, \infty) \times \mathbb{R}^{2}$, so that

$$
\begin{aligned}
\dot{W}_{(2.2)}(t, x, y)=- & |p(t)| e^{-P(t)} V(t, x, y)+e^{-P(t)} \dot{V}_{(2.2)}(t, x, y) \\
\leq- & \frac{|p(t)|}{2} e^{2 E(t)-P(t)}\left(x^{2}+\frac{f(t)}{g(t)} y^{2}\right)-\frac{f(t)}{2 g(t)} e^{2 E(t)-P(t)} \psi(t) y^{2} \\
& \quad+\frac{f(t)|p(t)|}{g(t)} e^{2 E(t)-P(t)}|y| \\
= & -\frac{f(t)}{2 g(t)} e^{2 E(t)-P(t)} \psi(t) y^{2}-\frac{|p(t)|}{2} e^{2 E(t)-P(t)} \\
& \times\left(x^{2}+\frac{f(t)}{g(t)}\left(y^{2}-2|y|\right)\right) \\
\leq- & \frac{f(t)}{2 g(t)} e^{2 E(t)-P(t)} \psi(t) y^{2}+\frac{f(t)|p(t)|}{2 g(t)} e^{2 E(t)-P(t)} \\
\leq & \frac{f(t)}{2 g(t)} e^{2 E(t)-P(t)} \psi-(t) y^{2}+\frac{f(t)|p(t)|}{2 g(t)} e^{2 E(t)-P(t)}
\end{aligned}
$$

Let $\chi(t)=\frac{f(t)|p(t)|}{2 g(t)} e^{2 E(t)-P(t)}$. Then,

$$
\dot{W}_{(2.2)}(t, x, y) \leq \frac{f(t)}{2 g(t)} e^{2 E(t)-P(t)} \psi_{-}(t) y^{2}+\chi(t) \leq \psi_{-}(t) W(t, x, y)+\chi(t) .
$$

Take

$$
U(t, x, y)=\exp \left(-\int_{t_{0}}^{t} \psi_{-}(s) d s\right)\left(W(t, x, y)+\int_{t}^{\infty} \chi(s) d s\right)
$$

on $[0, \infty) \times \mathbb{R}^{2}$, so that

$$
\begin{aligned}
\dot{U}_{(2.2)}(t, x, y)=- & \psi_{-}(t) U(t, x, y)+\exp \left(-\int_{t_{0}}^{t} \psi_{-}(s) d s\right) \\
& \times\left(\dot{W}_{(2.2)}(t, x, y)-\chi(t)\right) \\
= & -\psi_{-}(t) U(t, x, y)+\psi_{-}(t) \exp \left(-\int_{t_{0}}^{t} \psi_{-}(s) d s\right) W(t, x, y) .
\end{aligned}
$$

Since $\chi(t) \geq 0$ for $t \geq 0$, we obtain $\dot{U}_{(2.2)}(t, x, y) \leq 0$ on $[0, \infty) \times \mathbb{R}^{2}$. 
From assumptions (i), (vi) and (v), we can find positive numbers $L, M$ and $N$ such that

$$
E(t)>-L, \quad P(t)<M \quad \text { and } \quad \int_{0}^{t} \psi_{-}(s) d s<N
$$

for $t \geq 0$, respectively. Hence, by assumption (ii) and Lemma 2.2,

$$
V(t, x, y) \geq \frac{1}{2}\left(e^{-2 L} x^{2}+k y^{2}\right)
$$

on $[0, \infty) \times \mathbb{R}^{2}$ and, therefore,

$$
\begin{aligned}
& W(t, x, y) \geq e^{-M} V(t, x, y) \geq \frac{e^{-M}}{2}\left(e^{-2 L} x^{2}+k y^{2}\right) \\
& U(t, x, y) \geq e^{-N}\left(W(t, x, y)+\int_{t}^{\infty} \chi(s) d s\right) \geq \frac{e^{-M-N}}{2}\left(e^{-2 L} x^{2}+k y^{2}\right)
\end{aligned}
$$

on $[0, \infty) \times \mathbb{R}^{2}$.

Thus, $\dot{U}_{(2.2)}(t, x, y)$ is nonpositive and $U(t, x, y)$ tends to $\infty$ as $|x|+|y| \rightarrow \infty$ uniformly for $t \geq 0$. By means of a classical Lyapunov's direct method, we conclude that all solutions of (2.2) are equi-bounded (as to the direct method of Lyapunov, see, for example, [2, 3, 7-9, 12]).

We are now ready to move on to the main subject of this paper.

THEOREM 2.5. Suppose that assumptions (i)-(vi) are satisfied. Then, every solution $(x(t), y(t))$ of (2.2) tends to $(0,0)$ as $t \rightarrow \infty$.

PROOF. Recall that assumption (ii) yields the inequality (2.3) in Lemma 2.2. It follows from Lemma 2.4 that for any $t_{0} \geq 0$ and $\alpha>0$, there exists a $\beta\left(t_{0}, \alpha\right)>0$ such that $\left|x_{0}\right|+\left|y_{0}\right|<\alpha$ implies

$$
\left|x\left(t ; t_{0}, x_{0}, y_{0}\right)\right|+\left|y\left(t ; t_{0}, x_{0}, y_{0}\right)\right|<\beta \quad \text { for } t \geq t_{0} .
$$

For the sake of brevity, we write $(x(t), y(t))=\left(x\left(t ; t_{0}, x_{0}, y_{0}\right), y\left(t ; t_{0}, x_{0}, y_{0}\right)\right)$ and denote

$$
u(t)=\frac{f(t)}{2 g(t)} e^{2 E(t)} y^{2}(t) \quad \text { and } \quad v(t)=V(t, x(t), y(t)) .
$$

Then,

$$
v(t)=\frac{1}{2} e^{2 E(t)}\left(x^{2}(t)+\frac{f(t)}{g(t)} y^{2}(t)\right)=\frac{1}{2} e^{2 E(t)} x^{2}(t)+u(t)
$$

and

$$
v^{\prime}(t)=-\psi(t) u(t)+\frac{f(t) p(t)}{g(t)} e^{2 E(t)} y(t)
$$


for $t \geq t_{0}$. Hence, together with (2.3) and (2.7), we can estimate

$$
v^{\prime}(t) \leq \psi_{-}(t) v(t)+K|p(t)||y(t)| \leq \psi_{-}(t) v(t)+K \beta|p(t)|
$$

for $t \geq t_{0}$. Let $\phi(t)=K \beta|p(t)|$. Then, $\phi(t)$ is nonnegative for $t \geq t_{0}$. From assumption (vi), $\phi(t)$ is integrable on $\left[t_{0}, \infty\right)$. Since $v(t)$ is nonnegative and piecewise continuously differentiable for $t \geq t_{0}$, it follows from Lemma 2.3 that $v(t)$ has a limiting value $v_{0} \geq 0$. By (2.3), (2.8) and assumption (i),

$$
v(t) \geq \frac{1}{2} e^{-2 L} x^{2}(t)+\frac{k}{2} y^{2}(t)
$$

for some $L>0$. Hence, if $v_{0}=0$, then the solution $(x(t), y(t))$ tends to $(0,0)$ as $t \rightarrow \infty$. This completes the proof. Hereafter, we consider only the case in which $v_{0}>0$.

From (2.3) and (2.7), we see that $0 \leq u(t) \leq K \beta^{2} / 2$ for $t \geq t_{0}$, namely, $u(t)$ is bounded. Hence, $u(t)$ has an inferior limit and a superior limit. First, we shall show that the inferior limit of $u(t)$ is zero, and we shall then show that the superior limit of $u(t)$ is also zero.

Suppose that $\liminf _{t \rightarrow \infty} u(t)>0$. Then, there exist a $\gamma>0$ and a $T_{1} \geq t_{0}$ such that $u(t)>\gamma$ for $t \geq T_{1}$. From (2.9), it follows that

$$
\left|v^{\prime}(t)\right| \geq|\psi(t)| u(t)-\frac{f(t)|p(t)|}{g(t)} e^{2 E(t)}|y(t)|
$$

for $t \geq t_{0}$. Hence, using (2.3) and (2.7), again, we obtain

$$
\begin{aligned}
\int_{t_{0}}^{\infty}\left|v^{\prime}(s)\right| d s & \geq \int_{t_{0}}^{\infty}|\psi(s)| u(s) d s-\int_{t_{0}}^{\infty} \frac{f(s)|p(s)|}{g(s)} e^{2 E(s)}|y(s)| d s \\
& \geq \int_{t_{0}}^{\infty} \psi_{+}(s) u(s) d s-K \beta \int_{t_{0}}^{\infty}|p(s)| d s \\
& >\gamma \int_{T_{1}}^{\infty} \psi_{+}(s) d s-K \beta \int_{t_{0}}^{\infty}|p(s)| d s .
\end{aligned}
$$

Using Lemma 2.3 and assumption (vi),

$$
\infty>\int_{t_{0}}^{\infty}\left|v^{\prime}(s)\right| d s+K \beta \int_{t_{0}}^{\infty}|p(s)| d s>\gamma \int_{T_{1}}^{\infty} \psi_{+}(s) d s,
$$

which is a contradiction. Thus, we see that $\lim _{\inf } t \rightarrow \infty u(t)=0$.

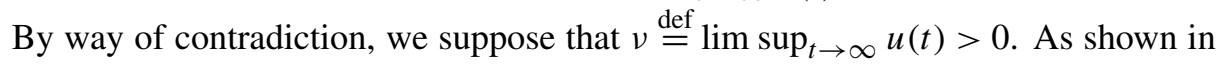
the proof of Lemma 2.2, we can choose a number $c_{4}>0$ satisfying

$$
\frac{|g(t)|}{e^{E(t)}} \geq c_{4} \quad \text { for } t \geq 0 .
$$


It also follows from assumption (iii) that there exists a $c_{5}>0$ with

$$
|h(t)| \leq c_{5} \quad \text { for } t \geq 0 .
$$

Since $v(t)$ tends to a positive value $v_{0}$ as $t \rightarrow \infty$, there exists $T_{2} \geq t_{0}$ such that

$$
0<\frac{v_{0}}{2}<v(t)<\frac{3 v_{0}}{2} \quad \text { for } t \geq T_{2} .
$$

Let $\varepsilon>0$ be so small that $\varepsilon<v / 2$ and

$$
c_{5} \sqrt{\frac{2 \varepsilon}{k}}<c_{4} \sqrt{v_{0}-2 \varepsilon}
$$

and let $c_{6}=c_{4} \sqrt{v_{0}-2 \varepsilon}-c_{5} \sqrt{2 \varepsilon / k}>0$. From assumption (vi), it follows that

$$
\int_{T_{3}}^{\infty}|p(s)| d s<\varepsilon
$$

for some $T_{3} \geq T_{2}$. Since $\liminf \inf _{t \rightarrow \infty} u(t)=0<v=\lim \sup _{t \rightarrow \infty} u(t)$, we can find two divergent sequences $\left\{\tau_{n}\right\}$ and $\left\{\sigma_{n}\right\}$ with $T_{3}<\tau_{n}<\sigma_{n}<\tau_{n+1}$ such that $u\left(\tau_{n}\right)=u\left(\sigma_{n}\right)=\varepsilon$,

$$
\begin{array}{ll}
u(t) \geq \varepsilon \quad \text { for } \tau_{n}<t<\sigma_{n}, \\
0 \leq u(t) \leq \varepsilon \quad \text { for } \sigma_{n}<t<\tau_{n+1}
\end{array}
$$

From (2.3), we see that $u(t) \geq k y^{2}(t) / 2$ for $t \geq t_{0}$. Hence, together with (2.14),

$$
|y(t)| \leq \sqrt{\frac{2}{k} u(t)}<\sqrt{\frac{2 \varepsilon}{k}} \quad \text { for } \sigma_{n} \leq t \leq \tau_{n+1} .
$$

By (2.8), (2.12) and (2.14), we obtain

$$
e^{E(t)}|x(t)|=\sqrt{2(v(t)-u(t))}>\sqrt{v_{0}-2 \varepsilon}
$$

for $\sigma_{n} \leq t \leq \tau_{n+1}$. Since $x(t)$ is continuous, we see that

$$
e^{E(t)} x(t)>\sqrt{v_{0}-2 \varepsilon} \text { or } e^{E(t)} x(t)<-\sqrt{v_{0}-2 \varepsilon}
$$

for $\sigma_{n} \leq t \leq \tau_{n+1}$. In addition, since $g(t)$ is continuous for $t \geq 0$, it follows from (2.10) that

$$
\frac{g(t)}{e^{E(t)}} \geq c_{4} \quad \text { or } \quad \frac{g(t)}{e^{E(t)}} \leq-c_{4}
$$

for $t \geq 0$. Hence, there are two cases to consider: (a) $g(t) x(t)>c_{4} \sqrt{v_{0}-2 \varepsilon}$ for $\sigma_{n} \leq t \leq \tau_{n+1}$ and (b) $g(t) x(t)<-c_{4} \sqrt{v_{0}-2 \varepsilon}$ for $\sigma_{n} \leq t \leq \tau_{n+1}$. 
In the former, from (2.11) and (2.15) and the second equation of (2.2), we can estimate that

$$
\begin{aligned}
y^{\prime}(t) & <-c_{4} \sqrt{v_{0}-2 \varepsilon}+c_{5}|y(t)|+|p(t)| \\
& \leq-c_{4} \sqrt{v_{0}-2 \varepsilon}+c_{5} \sqrt{\frac{2 \varepsilon}{k}}+|p(t)|=-c_{6}+|p(t)|
\end{aligned}
$$

for $\sigma_{n} \leq t \leq \tau_{n+1}$. Integrate this inequality from $\sigma_{n}$ to $\tau_{n+1}$ to obtain

$$
\left|y\left(\tau_{n+1}\right)\right|+\left|y\left(\sigma_{n}\right)\right|+\int_{\sigma_{n}}^{\tau_{n+1}}|p(s)| d s>c_{6}\left(\tau_{n+1}-\sigma_{n}\right) .
$$

Hence, from (2.7) and (2.13), we see that

$$
\tau_{n+1}<\sigma_{n}+\Delta
$$

where $\Delta=(\varepsilon+2 \beta) / c_{6}>0$. In case $(\mathrm{b})$,

$$
\begin{aligned}
y^{\prime}(t) & >c_{4} \sqrt{v_{0}-2 \varepsilon}-c_{5}|y(t)|-|p(t)| \\
& \geq c_{4} \sqrt{v_{0}-2 \varepsilon}-c_{5} \sqrt{\frac{2 \varepsilon}{k}}-|p(t)|=c_{6}-|p(t)|
\end{aligned}
$$

for $\sigma_{n} \leq t \leq \tau_{n+1}$. We obtain the inequality (2.16) in the above-described manner. Let $I=\bigcup_{n=1}^{\infty}\left[\tau_{n}, \sigma_{n}\right]$. By means of Lemma 2.3 with (2.3), (2.7), (2.14) and assumption (vi), again

$$
\infty>\int_{t_{0}}^{\infty}\left|v^{\prime}(s)\right| d s+K \beta \int_{t_{0}}^{\infty}|p(s)| d s>\int_{t_{0}}^{\infty} \psi_{+}(s) u(s) d s>\varepsilon \int_{I} \psi_{+}(s) d s .
$$

Hence, from (2.16) and assumption (iv), we see that

$$
\liminf _{n \rightarrow \infty}\left(\sigma_{n}-\tau_{n}\right)=0
$$

Since $\liminf _{t \rightarrow \infty} u(t)=0$ and $\limsup _{t \rightarrow \infty} u(t)=v>0$, we can choose two sequences $\left\{t_{n}\right\}$ and $\left\{s_{n}\right\}$ with $T_{3}<t_{n}<s_{n}<t_{n+1}$ such that $u\left(t_{n}\right)=v / 2, u\left(s_{n}\right)=3 v / 4$ and

$$
\frac{v}{2}<u(t)<\frac{3 v}{4} \text { for } t_{n}<t<s_{n} .
$$

Since $\varepsilon<v / 2$, we may consider that $\left[t_{n}, s_{n}\right] \subset\left[\tau_{n}, \sigma_{n}\right]$ for $n \in \mathbb{N}$ (if necessary, we can change $\left\{\tau_{n}\right\}$ and $\left\{\sigma_{n}\right\}$ into suitable subsequences of $\left\{\tau_{n}\right\}$ and $\left.\left\{\sigma_{n}\right\}\right)$. It follows naturally from (2.18) that

$$
\liminf _{n \rightarrow \infty}\left(s_{n}-t_{n}\right)=0 .
$$

As shown in Lemma 2.2, assumption (ii) implies (2.5). Since

$$
e^{E(t)}|x(t)|=\sqrt{2(v(t)-u(t))} \quad \text { for } t \geq t_{0},
$$


by (2.12) and (2.18),

$$
e^{E(t)}|x(t)|<\sqrt{3 v_{0}-v} \text { for } t_{n} \leq t \leq s_{n} .
$$

Hence, together with (2.5) and (2.7), we obtain

$$
\begin{aligned}
u^{\prime}(t) & =v^{\prime}(t)-e(t) e^{2 E(t)} x^{2}(t)-e^{2 E(t)} x(t) x^{\prime}(t) \\
& =v^{\prime}(t)-e(t) e^{2 E(t)} x^{2}(t)-e^{2 E(t)} x(t)(-e(t) x(t)+f(t) y(t)) \\
& \leq\left|v^{\prime}(t)\right|+|f(t)| e^{E(t)} e^{E(t)}|x(t)||y(t)| \\
& <\left|v^{\prime}(t)\right|+c_{3} \beta \sqrt{3 v_{0}-v}
\end{aligned}
$$

for $t_{n} \leq t \leq s_{n}$. Integrating this inequality from $t_{n}$ to $s_{n}$, we obtain

$$
\frac{v}{4}=\left|u\left(s_{n}\right)\right|-\left|u\left(t_{n}\right)\right| \leq \int_{t_{n}}^{s_{n}}\left|v^{\prime}(s)\right| d s+c_{3} \beta \sqrt{3 v_{0}-v}\left(s_{n}-t_{n}\right)
$$

for each $n \in \mathbb{N}$. By (2.19), the right-hand side of the above inequality tends to zero as $n \rightarrow \infty$. This is a contradiction. We therefore conclude that $\lim _{\sup } \operatorname{su}_{t \rightarrow \infty} u(t)=0$.

In summary, $u(t)$ tends to zero as $t \rightarrow \infty$. Hence, there exists a $T_{4} \leq t_{0}$ such that

$$
u(t)<\varepsilon \quad \text { for } t \geq T_{4} .
$$

Using this estimation instead of (2.14) and following the same process as in the preceding paragraph, we see that

$$
y^{\prime}(t)<-c_{6}+|p(t)| \quad \text { or } \quad y^{\prime}(t)>c_{6}-|p(t)|
$$

for $t \geq T_{4}$. In the former case,

$$
y(t)-y\left(T_{4}\right)<-c_{6}\left(t-T_{4}\right)+\int_{T_{4}}^{t}|p(s)| d s \rightarrow-\infty \quad \text { as } t \rightarrow \infty .
$$

This contradicts (2.7). In the latter case,

$$
y(t)-y\left(T_{4}\right)>c_{6}\left(t-T_{4}\right)-\int_{T_{4}}^{t}|p(s)| d s \rightarrow \infty \quad \text { as } t \rightarrow \infty,
$$

which is a contradiction. Thus, the case of $v_{0}>0$ does not occur.

The proof of Theorem 2.5 is thus complete.

To show a simple example of application, we consider the self-adjoint differential equation

$$
\left(a(t) x^{\prime}\right)^{\prime}+b(t) x^{\prime}+c(t) x=p(t),
$$

where $a(t)$ and $c(t)$ are continuously differentiable for $t \geq 0, a(t) c(t)>0$ for $t \geq 0$, and $b(t)$ and $p(t)$ are continuous for $t \geq 0$. We can rewrite this equation as an equivalent system:

$$
x^{\prime}=\frac{1}{a(t)} y, \quad y^{\prime}=-c(t) x-\frac{b(t)}{a(t)} x+p(t),
$$


which has the form of (2.2) with $e(t)=0, \quad f(t)=1 / a(t), \quad g(t)=c(t)$ and $h(t)=b(t) / a(t)$. Judging from this situation, it is valuable to state the case that $e(t)=0$ as a corollary. Since $e(t)$ vanishes, $E(t)$ is equal to zero. Hence, assumption (ii) becomes

(vii) $f(t)$ and $g(t)$ are bounded, $f(t) g(t)>0$ for $t \geq 0$ and $\liminf _{t \rightarrow \infty} f(t) g(t)>0$.

Corollary 2.6. Suppose that $e(t)=0$ for $t \geq 0$ and assumptions (iii)-(vii) are satisfied. Then, every solution $(x(t), y(t))$ of $(2.2)$ tends to $(0,0)$ as $t \rightarrow \infty$.

Let $z=e^{E(t)} x$ and $w=e^{E(t)} y$. Then, system (2.2) is transformed into the system

$$
z^{\prime}=f(t) w, \quad w^{\prime}=-g(t) z-\tilde{h}(t) w+e^{E(t)} p(t),
$$

where $\widetilde{h}(t)=h(t)-e(t)$. It is clear that

$$
\widetilde{\psi}(t)=2 \tilde{h}(t)+\frac{f(t)}{g(t)}\left(\frac{g(t)}{f(t)}\right)^{\prime}=2(h(t)-e(t))+\frac{f(t)}{g(t)}\left(\frac{g(t)}{f(t)}\right)^{\prime}=\psi(t) .
$$

Under assumption (i), every solution $(x(t), y(t))$ of (2.2) tends to $(0,0)$ as $t \rightarrow \infty$ if and only if the corresponding solution $(z(t), w(t))$ of $(2.20)$ tends to $(0,0)$ as $t \rightarrow \infty$. Hence, Corollary 2.6 yields the following results.

THEOREM 2.7. Suppose that assumptions (i), (iv), (v) and (vii) are satisfied and suppose that:

(viii) $h(t)-e(t)$ is bounded;

(ix) $\int_{0}^{\infty} e^{E(s)}|p(s)| d s<\infty$.

Then, every solution of (2.2) tends to $(0,0)$ as $t \rightarrow \infty$.

COROllary 2.8. Suppose that assumptions (i), (iv), (v) and (vii)-(ix) are satisfied. Then, the zero solution of (2.1) is asymptotically stable. If, in addition, $E(t)$ is bounded from above, then the zero solution of (2.1) is uniformly stable.

\section{Perturbed system}

In this section, we deal with perturbation problems on the convergence of solutions. For this purpose, we consider the perturbed system

$$
x^{\prime}=-e(t) x+f(t) y, \quad y^{\prime}=-g(t) x-h(t) y+q(t, x, y),
$$

where $f(t)$ and $g(t)$ are continuous for $t \geq 0, e(t)$ and $h(t)$ are piecewise continuous for $t \geq 0$, and $q(t, x, y)$ is continuous for $t \geq 0$ and $(x, y) \in \mathbb{R}^{2}$.

DEFINITION 3.1. A continuous function $q$ is said to be integrable on disks if for every $H>0$, there exists a function $p_{H}$ such that

$$
|q(t, x, y)| \leq p_{H}(t) \text { for } t \geq 0 \text { and }(x, y) \in D_{H},
$$

where $\int_{0}^{\infty} p_{H}(s) d s<\infty$ and $D_{H}=\{(x, y):|x|+|y|<H\}$. 
For example, if $q(t, x, y)=p(t) \exp (|x|+|y|)$ for $t \geq 0$ and $(x, y) \in \mathbb{R}^{2}$, where $p(t)$ satisfies assumption (vi) of Lemma 2.4, then $q(t, x, y)$ is integrable on disks.

We again consider systems $(L)$ and $(P)$ given in Section 1 . Let $Y(t)$ be a fundamental matrix for system $(L)$. Then, the zero solution of $(L)$ is uniformly stable if and only if $\left\|Y(t) Y^{-1}(s)\right\|$ is bounded for $0 \leq s \leq t \leq \infty$ and is asymptotically stable if and only if $\|Y(t)\|$ tends to 0 as $t \rightarrow \infty$. In [1, Theorem 6, p. 64], Coppel has shown that if $Y(t)$ satisfies the two properties above and if

$$
\|\mathbf{f}(t, \mathbf{x})\| \leq \lambda(t)\|\mathbf{x}\|
$$

for $t \geq 0$ and $\mathbf{x} \in \mathbb{R}^{n}$, where $\int_{0}^{\infty} \lambda(s) d s<\infty$, then every solution $\mathbf{x}(t)$ of $(P)$ tends to $\mathbf{0}$ as $t \rightarrow \infty$. In other words, under assumption (3.2), if the zero solution of $(L)$ is uniformly stable and asymptotically stable, then every solution of $(P)$ approaches the origin $\mathbf{0}$ as $t$ increases. In general, it is difficult to seek a concrete fundamental matrix $Y(t)$ for system $(L)$, but fortunately we can check the uniform stability and the asymptotic stability of the zero solution of (2.1).

By virtue of Coppel's result and Theorem A, we see that if assumptions (i)-(v) are satisfied and if

$$
|q(t, x, y)| \leq \lambda(t)(|x|+|y|) \quad \text { for } t \geq 0 \text { and }(x, y) \in \mathbb{R}^{2},
$$

where $\lambda(t)$ is an integrable function, then every solution $(x(t), y(t))$ of (3.1) tends to $(0,0)$ as $t \rightarrow \infty$. Of course, if $q(t, x, y)$ satisfies condition (3.3), then it is integrable on disks. The converse is false, because $\exp (|x|+|y|) /(1+t)$ is integrable on disks.

Now, under the assumption that $q(t, x, y)$ is integrable on disks, does every solution of (3.1) converge to the origin $(0,0)$ ? Before answering this question, the boundedness of solutions of (3.1) must be clarified. As used in the proof of Lemma 2.4, a basic Lyapunov theorem for the boundedness of solutions of planar systems is as follows. If there exists a continuous function $U:[0, \infty) \times \mathbb{R}^{2} \rightarrow[0, \infty)$ that is locally Lipschitz in $(x, y)$, if $U(t, x, y) \rightarrow \infty$ as $|x|+|y| \rightarrow \infty$ uniformly for $t \geq 0$, and if the derivative of $U$ along any solution is nonpositive on $[0, \infty) \times \mathbb{R}^{2}$, then all solutions are equi-bounded. It is easy to weaken the assumption on the derivative of $U$, as follows:

$$
\dot{U}(t, x, y) \leq 0 \quad \text { on }[0, \infty) \times D_{H}
$$

for any $H>0$.

LEMMA 3.2. Suppose that assumptions (i), (ii) and (v) hold. If $q(t, x, y)$ is integrable on disks, then all solutions of (3.1) are equi-bounded.

PROOF. Define

$$
V(t, x, y)=\frac{1}{2} e^{2 E(t)}\left(x^{2}+\frac{f(t)}{g(t)} y^{2}\right),
$$


so that

$$
\dot{V}_{(3.1)}(t, x, y)=-\frac{f(t)}{2 g(t)} e^{2 E(t)} \psi(t) y^{2}+\frac{f(t)}{g(t)} e^{2 E(t)} q(t, x, y) y .
$$

For any $H>0$, let $P_{H}(t)=\int_{0}^{t} p_{H}(s) d s$. Now consider $W(t, x, y)=e^{-P_{H}(t)} V$ $(t, x, y)$ and find the derivative of $W$ by computing

$$
\begin{aligned}
\dot{W}_{(3.1)}(t, x, y)= & -p_{H}(t) e^{-P_{H}(t)} V(t, x, y)+e^{-P_{H}(t)} \dot{V}_{(3.1)}(t, x, y) \\
\leq & -\frac{p_{H}(t)}{2} e^{2 E(t)-P_{H}(t)}\left(x^{2}+\frac{f(t)}{g(t)} y^{2}\right)-\frac{f(t)}{2 g(t)} e^{2 E(t)-P_{H}(t)} \psi(t) y^{2} \\
& \quad+\frac{f(t) p_{H}(t)}{g(t)} e^{2 E(t)-P_{H}(t)}|y| \\
= & -\frac{f(t)}{2 g(t)} e^{2 E(t)-P_{H}(t)} \psi(t) y^{2}-\frac{p_{H}(t)}{2} e^{2 E(t)-P_{H}(t)} \\
& \times\left(x^{2}+\frac{f(t)}{g(t)}\left(y^{2}-2|y|\right)\right) \\
\leq- & \frac{f(t)}{2 g(t)} e^{2 E(t)-P_{H}(t)} \psi(t) y^{2}+\frac{f(t) p_{H}(t)}{2 g(t)} e^{2 E(t)-P_{H}(t)} \\
\leq & \frac{f(t)}{2 g(t)} e^{2 E(t)-P_{H}(t)} \psi_{-}(t) y^{2}+\frac{f(t) p_{H}(t)}{2 g(t)} e^{2 E(t)-P_{H}(t)}
\end{aligned}
$$

for $t \geq 0$ and $(x, y) \in D_{H}$. Let $\chi(t)=\frac{f(t) p_{H}(t)}{2 g(t)} e^{2 E(t)-P_{H}(t)}$. Then, $\chi(t)>0$ for $t \geq 0$. Next, we define

$$
U(t, x, y)=\exp \left(-\int_{t_{0}}^{t} \psi_{-}(s) d s\right)\left(W(t, x, y)+\int_{t}^{\infty} \chi(s) d s\right)
$$

and obtain

$$
\begin{aligned}
\dot{U}_{(3.1)}(t, x, y) & =-\psi_{-}(t) U(t, x, y)+\exp \left(-\int_{t_{0}}^{t} \psi_{-}(s) d s\right)\left(\dot{W}_{(3.1)}(t, x, y)-\chi(t)\right) \\
& =-\psi_{-}(t) U(t, x, y)+\psi_{-}(t) \exp \left(-\int_{t_{0}}^{t} \psi_{-}(s) d s\right) W(t, x, y) \leq 0
\end{aligned}
$$

on $[0, \infty) \times D_{H}$.

From assumptions (i) and (v) and Definition 3.1, we can choose positive numbers $L, M$ and $N$ with

$$
E(t)>-L, \quad P_{H}(t)<M \quad \text { and } \quad \int_{0}^{t} \psi_{-}(s) d s<N
$$

for $t \geq 0$. Hence, by assumption (ii) and Lemma 2.2,

$$
U(t, x, y) \geq e^{-N}\left(W(t, x, y)+\int_{t}^{\infty} \chi(s) d s\right) \geq \frac{e^{-M-N}}{2}\left(e^{-2 L} x^{2}+k y^{2}\right) .
$$


Since $U(t, x, y)$ tends to $\infty$ as $|x|+|y| \rightarrow \infty$ uniformly for $t \geq 0$ and $\dot{U}_{(3.1)}(t, x, y)$ is nonpositive on $[0, \infty) \times D_{H}$ for any $H>0$, we conclude that all solutions of (3.1) are equi-bounded.

Lemma 3.2 guarantees that for any $t_{0} \geq 0$ and $\alpha>0$, there exists a $\beta\left(t_{0}, \alpha\right)>0$ such that $\left|x_{0}\right|+\left|y_{0}\right|<\alpha$ implies

$$
\left|x\left(t ; t_{0}, x_{0}, y_{0}\right)\right|+\left|y\left(t ; t_{0}, x_{0}, y_{0}\right)\right|<\beta \quad \text { for } t \geq t_{0} .
$$

For simplicity, let $(x(t), y(t))=\left(x\left(t ; t_{0}, x_{0}, y_{0}\right), y\left(t ; t_{0}, x_{0}, y_{0}\right)\right)$. If $q(t, x, y)$ is integrable on disks, then

$$
|q(t, x(t), y(t))| \leq p_{\beta}(t) \quad \text { for } t \geq 0 .
$$

Let

$$
u(t)=\frac{f(t)}{2 g(t)} e^{2 E(t)} y^{2}(t) \quad \text { and } \quad v(t)=V(t, x(t), y(t)) .
$$

Then, we obtain

$$
v(t)=\frac{1}{2} e^{2 E(t)}\left(x^{2}(t)+\frac{f(t)}{g(t)} y^{2}(t)\right)=\frac{1}{2} e^{2 E(t)} x^{2}(t)+u(t)
$$

and

$$
\begin{aligned}
v^{\prime}(t) & =-\psi(t) u(t)+\frac{f(t)}{g(t)} e^{2 E(t)} q(t, x(t), y(t)) y(t) \\
& \leq \psi_{-}(t) v(t)+\frac{f(t)}{g(t)} e^{2 E(t)} p_{\beta}(t)|y(t)| \leq \psi_{-}(t) v(t)+\beta \frac{f(t)}{g(t)} e^{2 E(t)} p_{\beta}(t)
\end{aligned}
$$

for $t \geq t_{0}$. Hence, using $p_{\beta}(t)$ instead of $|p(t)|$, we can proceed with the same argument as in the proof of Theorem 2.5 (we omit the details).

THEOREM 3.3. Suppose that assumptions (i)-(v) are satisfied. If $q(t, x, y)$ is integrable on disks, then every solution $(x(t), y(t))$ of (3.1) tends to $(0,0)$ as $t \rightarrow \infty$.

In [1, Theorem 6, p. 64], we need both uniform stability and asymptotic stability of the zero solution of (2.1) to show that every solution of (3.1) converges to the origin $(0,0)$. On the other hand, in Theorem 3.3, uniform stability of the zero solution of (2.1) is unnecessary for the convergence of solutions of (3.1).

To compare Theorem 3.3 with [1, Theorem 6, p. 64], we present some simple examples. The linear equation

$$
x^{\prime \prime}+\frac{2}{1+t} x^{\prime}+x=0
$$

is equivalent to system (2.1) with $e(t)=0, f(t)=g(t)=1$ and $h(t)=2 /(1+t)$. As mentioned in Section 2, from Theorem A we see that the equilibrium $\left(x, x^{\prime}\right)=(0,0)$ of (3.4) is uniformly stable and asymptotically stable. Let us add a forcing term to equation (3.4). 
EXAMPLE 3.4. Consider the equation

$$
x^{\prime \prime}+\frac{2}{1+t} x^{\prime}+x=\frac{\exp \left(|x|+\left|x^{\prime}\right|\right)}{1+t} .
$$

Then, every solution $x(t)$ of (3.5) as well as its derivative $x^{\prime}(t)$ tends to zero as $t \rightarrow \infty$.

Letting $y=x^{\prime}$, equation (3.5) becomes the system

$$
x^{\prime}=y, \quad y^{\prime}=-x-\frac{2}{1+t} y+\frac{\exp (|x|+|y|)}{1+t} .
$$

Since $q(t, x, y)=\exp (|x|+|y|) /(1+t)$ in system (3.6), it is integrable on disks with $p_{H}(t)=e^{2 H} /(1+t)$. Hence, from Theorem 3.3 it turns out that every solution $(x(t), y(t))$ of (3.6) tends to $(0,0)$ as $t \rightarrow \infty$. However, this fact cannot be judged by Coppel's result, because $q(t, x, y)$ does not satisfy condition (3.3).

System (3.1) does not always have the zero solution, but if $q(t, 0,0)=0$, then system (3.1) has the zero solution. The functions

$$
W(t, x, y)=\frac{1}{2} e^{2 E(t)-P_{H}(t)}\left(x^{2}+\frac{f(t)}{g(t)} y^{2}\right)
$$

and

$$
U(t, x, y)=\exp \left(-\int_{t_{0}}^{t} \psi_{-}(s) d s\right)\left(W(t, x, y)+\int_{t}^{\infty} \chi(s) d s\right)
$$

are defined in the proof of Lemma 3.2. Recall that $U(t, x, y)$ is positive definite and its derivative $\dot{U}_{(3.1)}(t, x, y)$ is nonpositive for $t \geq 0$ and $(x, y)$ in the neighborhood of the origin $(0,0)$. From these properties, we see that the zero solution of (3.1) is stable. Hence, together with Theorem 3.3. we have the following result.

THEOREM 3.5. In addition to all of the assumptions in Theorem 3.3, if $q(t, 0,0)=0$, then the zero solution of (3.1) is asymptotically stable. Moreover, if $E(t)$ is bounded from above, then the zero solution of (3.1) is uniformly stable.

In Coppel's result described above, if condition (3.2) holds for $t \geq 0$ and $\mathbf{x}$ in a bounded domain of $\mathbb{R}^{n}$, then every solution $\mathbf{x}(t)$ of $(P)$ in the neighborhood of the origin $\mathbf{0}$ tends to $\mathbf{0}$ as $t \rightarrow \infty$, and the zero solution of $(P)$ is uniformly stable and asymptotically stable. If there exists no neighborhood of $\mathbf{0}$ satisfying condition (3.2), then Coppel's result is meaningless.

EXAMPLE 3.6. Consider the equation

$$
x^{\prime \prime}+\frac{2}{1+t} x^{\prime}+x=\frac{\sqrt{|x|}+\sqrt{\left|x^{\prime}\right|}}{1+t} .
$$

Then, the equilibrium $\left(x, x^{\prime}\right)=(0,0)$ of (3.7) is uniformly stable and asymptotically stable. 
The system

$$
x^{\prime}=y, \quad y^{\prime}=-x-\frac{2}{1+t} y+\frac{\sqrt{|x|}+\sqrt{|y|}}{1+t}
$$

is equivalent to (3.7). It is clear that assumptions (i)-(v) hold and $E(t) \equiv 0$. Since $q(t, x, y)=(\sqrt{|x|}+\sqrt{|y|}) /(1+t)$, it is integrable on disks with $p_{H}(t)=\sqrt{2 H} /(1+t)$ and $q(t, 0,0)=0$ for $t \geq 0$. Hence, from Theorem 3.5 we see that the zero solution of (3.8) is uniformly stable and asymptotically stable.

\section{References}

[1] W. A. Coppel, Stability and Asymptotic Behavior of Differential Equations (Heath, Boston, MA, 1965).

[2] A. Halanay, Differential Equations: Stability, Oscillations, Time Lags (Academic Press, New York, 1966).

[3] J. K. Hale, Ordinary Differential Equations (Wiley-Interscience, New York, 1969). (Revised, Krieger, Malabar, 1980).

[4] L. Hatvani, 'A generalization of the Barbashin-Krasovskij theorems to the partial stability in nonautonomous systems', in: Qualitative Theory of Differential Equations I, II, Colloquia Mathematica Societatis János Bolyai, 30 (ed. M. Farkas) (North-Holland, Amsterdam, 1981), pp. 381-409.

[5] $\longrightarrow$, 'On the uniform attractivity of solutions of ordinary differential equations by two Lyapunov functions', Proc. Japan Acad. 67 (1991), 162-167.

[6] $\longrightarrow$, 'On the asymptotic stability for a two-dimensional linear nonautonomous differential system', Nonlinear Anal. 25 (1995), 991-1002.

[7] J. P. LaSalle and S. Lefschetz, Stability by Liapunov's Direct Method with Applications, Mathematics in Science and Engineering, 4 (Academic Press, New York, 1961).

[8] D. R. Merkin, Introduction to the Theory of Stability, Texts in Applied Mathematics, 24 (Springer, New York, 1997).

[9] N. Rouche, P. Habets and M. Laloy, Stability Theory by Liapunov's Direct Method, Applied Mathematical Sciences, 22 (Springer, New York, 1977).

[10] A. Strauss and J. A. Yorke, 'Perturbing uniformly stable linear systems with and without attraction', SIAM J. Appl. Math. 17 (1969), 725-738.

[11] J. Sugie, 'Influence of anti-diagonals on the asymptotic stability for linear differential systems', accepted for Monatsh. Math. at press.

[12] T. Yoshizawa, Stability Theory by Liapunov's Second Method, Publications of the Mathematical Society of Japan, 9 (Mathematical Society of Japan, Tokyo, 1966).

JITSURO SUGIE, Department of Mathematics and Computer Science,

Shimane University, Matsue 690-8504, Japan

e-mail: jsugie@ riko.shimane-u.ac.jp 